\title{
Abundances anomalies and meridional circulation in horizontal branch stars
}

\author{
D. Quievy ${ }^{1}$, P. Charbonneau ${ }^{1}$, G. Michaud ${ }^{1,2}$, and J. Richer ${ }^{1}$ \\ 1 Département de Physique, Université de Montréal, Montréal, PQ, H3C 3J7, Canada \\ e-mail: [paulchar;michaudg; jacques.richer] @umontreal.ca \\ 2 LUTH, Observatoire de Paris, CNRS, Université Paris Diderot, 5 Place Jules Janssen, 92190 Meudon, France
}

Received 30 October 2008 / Accepted 16 February 2009

\begin{abstract}
Context. Photospheric chemical abundances on the horizontal branch (HB) show some striking variations with effective temperature $\left(T_{\text {eff }}\right)$. The most straightforward explanation is that these anomalies develop through diffusion processes, in particular gravitational settling and radiative levitation. However, the abrupt disappearance of strong abundance anomalies as one moves below about $11000 \mathrm{~K}$ on the HB suggests that another factor plays an important role.

Aims. We test an extension to the HB of the diffusion model for main-sequence $\mathrm{HgMn}$ stars, where strong anomalies can only develop in the slower rotators. In these rotators the gravitational settling of helium leads to the disappearance of its superficial convection zone, so that chemical separation by radiative levitation can occur all the way to the photosphere.

Methods. More specifically, we calculate the critical rotational velocity at which He settling is prevented by rotationally-induced meridional circulation, in a suite of stellar models spanning the zero-age HB. Helium settling serves as the measure of the atomic diffusion of all species.

Results. Our abundance evolution calculations show that, for models with $T_{\text {eff }}$ less than about $11500 \mathrm{~K}$, corresponding to stars typically observed with the same metal composition as giants, meridional circulation is efficient enough to suppress He settling for rotational velocities, in good agreement with observed values. Once the meridional circulation profile of a star rotating as a near rigid body has been adopted, no adjustable parameter is involved.

Conclusions. The $T_{\mathrm{eff}}$ dependence of abundance anomalies observed on the HB can be explained by atomic diffusion transport if one introduces the competition of meridional circulation with the observed $T_{\text {eff }}$ dependence of rotation velocity of HB stars.
\end{abstract}

Key words. diffusion - stars: abundances - stars: horizontal branch - stars: chemically peculiar - stars : interiors

\section{Astrophysical context}

The horizontal branch (HB) of globular clusters is believed to be populated by stars that have recently gone through an helium flash at the tip of the red giant branch (RGB). The He core mass, $M_{\mathrm{c}}$, is generally expected to be constant on the HB but the hydrogen envelope mass, $M_{\mathrm{env}}$, is believed to vary according to the mass loss that occurred around the tip of the RGB. The $M_{\text {env }}$ determines the effective temperature of the star on the HB. Many properties of HB stars vary with $T_{\text {eff }}$ and are only partially understood. They are reviewed for instance in Moehler (2001).

Since HB stars of a cluster have just evolved from the giant branch, they are all expected to start their HB evolution with approximately the same concentrations of metals, at least those heavier than Al. Nucleosynthesis may lead to variations in the concentration of CNO and other (relatively) light species, but iron peak elements are not expected to be substantially affected, as confirmed observationally (Gratton et al. 2004; Yong et al. 2008). However, on the HB, the iron peak abundance varies with $T_{\text {eff }}$ and this variation is coupled to rotational velocity variations. These abundance variations apparently must build up on the HB itself.

In all observed globular clusters containing $\mathrm{HB}$ stars with $T_{\text {eff }} \gtrsim 11000 \mathrm{~K}$, it was found that $[\mathrm{Fe} / \mathrm{H}],[\mathrm{Ti} / \mathrm{H}],[\mathrm{Mn} / \mathrm{H}]$, $[\mathrm{Cr} / \mathrm{H}]$, and $[\mathrm{P} / \mathrm{H}]$ have the same values as in giants below $\sim 11000 \pm 500 \mathrm{~K}$, while they are higher by factors $10-100$ for $T_{\text {eff }} \gtrsim 11000 \pm 500 \mathrm{~K}$ (Behr et al. 1999, 2000a; Behr 2003a; Moehler et al. 2000; Fabbian et al. 2005; Pace et al. 2006). Some elements, such as magnesium and silicon, remain nearly invariant, while He is underabundant when Fe peak elements are overabundant. The availability of larger telescopes thus confirmed earlier observations (Glaspey et al. 1989; Glaspey et al. 1986).

Progress has also been made in determining rotation velocities and their $T_{\text {eff }}$ dependence. Peterson (1983) noticed the $v \sin i$ diversity and its bimodal distribution - with $1 / 3$ of $v \sin i$ superior to $20 \mathrm{~km} \mathrm{~s}^{-1}$ for $T_{\text {eff }}$ lower than $10000 \mathrm{~K}$, and $2 / 3$ with lower $v \sin i$ for hotter stars. This trend has now been confirmed and made much more precise (Behr et al. 2000a,b; Recio-Blanco et al. 2002, 2004) with the addition of a link between abundance anomalies and rotation velocities. Stars with $T_{\text {eff }} \gtrsim 11000 \pm 500 \mathrm{~K}$ are slow rotators and have abundance anomalies, while those below this limit are fast rotators and have normal abundances (see Fig. 1).

That abundance anomalies of Fe peak elements should be observed in HB stars was originally suggested from theoretical considerations (Michaud et al. 1983). Their observation strongly suggests the role of some transport mechanism since nuclear reactions are not expected to modify their concentrations in that evolutionary stage. Then HB stars of globular clusters can become a laboratory for studying the role of hydrodynamical flows 


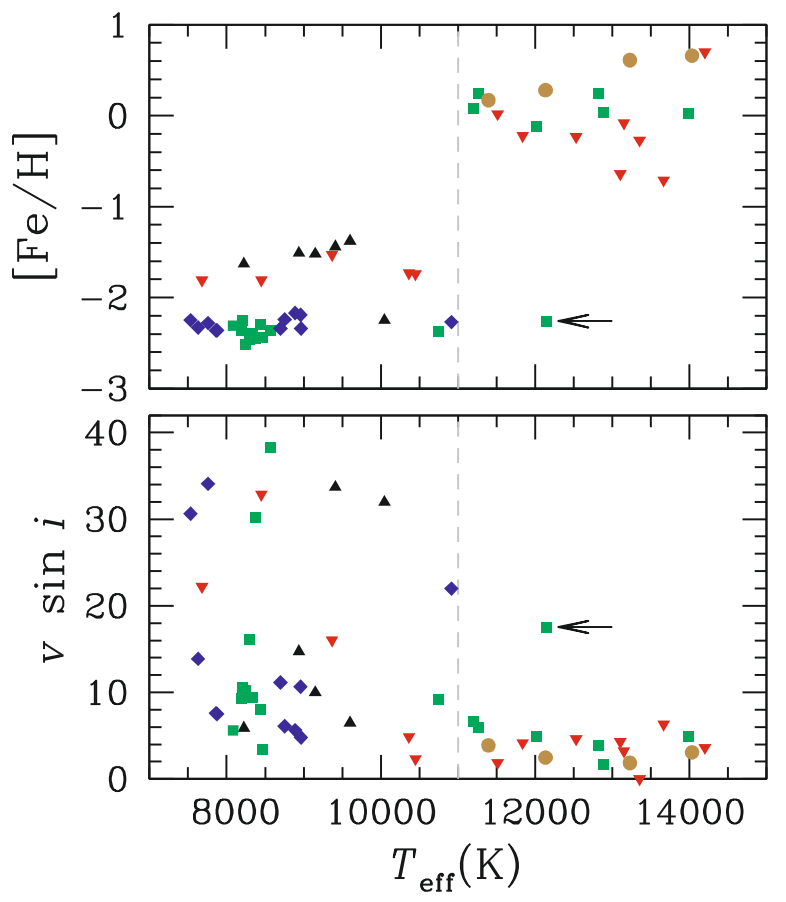

Fig. 1. In the upper part, $[\mathrm{Fe} / \mathrm{H}]$ as a function of $T_{\text {eff }}$ for all globular clusters in Behr (2003a) where all values of $[\mathrm{Fe} / \mathrm{H}]$ and $v \sin i$ are taken. Lower part, projected equatorial rotational velocity of the same stars. The different clusters are shape and color coded (black up-right triangles: M 3; red inverted triangles: M 13; green squares: M 15; blue diamonds: M 68; brown circles: NGC 288). There is a sharp boundary at $T_{\text {eff }}=11000 \mathrm{~K}$. All warmer stars have a high Feabundance except for the star B348 in M 15 (indicated by the arrow), which has a $v \sin i$ five times higher than other stars of similar $T_{\text {eff. }}$. Typical uncertainties in $T_{\text {eff }}$, as listed in Behr (2003a), range from around $\pm 100 \mathrm{~K}$ in the cooler stars, up to $\pm 500 \mathrm{~K}$ (and sometimes more) in the hotter stars; uncertainties in $v \sin i$ vary from about $\pm 1 \mathrm{~km} \mathrm{~s}^{-1}$ in the slow rotators, up to $\pm 5 \mathrm{~km} \mathrm{~s}^{-1}$ in the more rapidly rotating stars.

competing with atomic diffusion. Assume an unmagnetized atmosphere, mixed by a thin convection zone but assume, for the moment, that all other mixing processes are negligible and that there is no mass loss. Then, atomic diffusion at the bottom of the surface convection zone leads to underabundances in the convection zone, for species on which gravitational settling dominates, as for He, but to overabundances when radiative accelerations $\left(g_{\mathrm{rad}}\right)$ dominate, as for most Fe peak elements in HB stars (Michaud et al. 1983).

Numerous mixing processes may compete with atomic diffusion and reduce the abundance anomalies it leads to. Rotationally induced meridional circulation, mass loss, and turbulent diffusion may play a role, and turbulence itself may be produced in many ways in stellar interiors. While it is not currently possible to exclude any of those processes, we concentrate in this paper on the potential role of meridional circulation. The observed link between rotation and abundance anomalies suggests that the competing process is related to rotation, and meridional circulation is currently the only stellar hydrodynamical flow linked to rotation that can be described without arbitrary parameters.

A further link is suggested by observations. In the same part of the HR diagram as some HB stars, one finds HgMn stars. Those main sequence B stars owe their name to their peculiar high mercury and manganese concentrations (Jomaron et al. 1999; Woolf \& Lambert 1999). Here again, on the one hand, one has normal B stars with relatively fast rotation velocities, and on the other, $\mathrm{HgMn}$ stars, rotating slowly (Abt et al. 2002). They were first suggested to be related to the field star equivalent of HB stars by Sargent \& Searle $(1967,1968)$ on the basis of similar abundance anomalies in both types of stars. The link between rotation, meridional circulation and diffusion has already been suggested for HgMn stars. Above a critical value of the equatorial rotation velocity it has been shown and confirmed in 2D simulations (Michaud 1982; Charbonneau \& Michaud 1988, 1991) that meridional circulation could prevent gravitational settling from reducing the He concentration below the critical value for which the He convection zone disappears. It was also shown that the critical rotation velocity varies with gravity: from 90 to $4 \mathrm{~km} \mathrm{~s}^{-1}$ as $\log g$ goes from 4.4 to 3.5 . On the other hand, if the rotational velocity is lower than the critical value, gravitational settling reduces the He concentration, this reduces the opacity and affects the equation of state so that convection disappears. Chemical separation is then free to occur where there previously was a convection zone, and metal overabundances are reinforced. After this occurs, the high overabundances of $\mathrm{Mn}$ and $\mathrm{Hg}$ and other metals can materialize and many observed anomalies are explained in a parameter free model (Michaud 1986, 1981).

This does not imply that the meridional circulation model used is a complete description of the interior of rotating stars. The analytic solution of Tassoul \& Tassoul (1982, hereafter TT82) which was used was obtained under somewhat restrictive conditions. By using the meridional circulation obtained with the Tassoul model, one then tested to what extent it properly reproduces the most important transport modes of a complete simulation. While a complete 3-D simulation of meridional circulation in stars would be expected to require a large number of modes for a description of both the momentum and particle transport caused by the thermal imbalance (see Talon et al. 2003, for the description of a potential simulation; a critical review of 2-D simulations, e.g. Théado \& Vauclair 2003, and of meridional circulation models, e.g. Zahn 1992, is outside the scope of the present brief paper), particle transport might be mainly carried by the fundamental mode as is often the case in numerical simulations and in laboratory flows. One may interpret the success of the model in explaining HgMn stars as implying that the circulatory flows that TT82 obtain are a reasonable evaluation of the first order mode of a numerical simulation. We wish to test this possibility using HB stars.

Michaud et al. (2004) suggested that one may, for HB stars, use the same critical equatorial velocity as in $\mathrm{HgMn}$ stars where the HB crosses the main-sequencein the HR diagram. Then, applying the scaling expected from the results of Michaud (1982), one can determine the expected critical rotational velocity on the HB. In this way they obtained:

$v_{\mathrm{e}}^{l} \propto g^{1.75} / T_{\mathrm{eff}}^{2}$

By using the $100 \mathrm{~km} \mathrm{~s}^{-1}$ limit for HgMn stars of $T_{\text {eff }}=15000 \mathrm{~K}$ and $\log g=4.4$ one can anchor Eq. (1) and predict the limiting rotational velocity on the $\mathrm{HB}$ where both gravity and $T_{\text {eff }}$ change along the branch. Those authors obtained reasonable success with this simple model. The basic question examined in the present paper is then whether the change of structure from the main-sequenceto the HB allows to make such a prediction. If one repeats the calculations of Charbonneau \& Michaud (1988) for HB stars, will one obtain for the stellar structure of HB stars the same scaling law as obtained from main-sequencemodels? The structure of HB stars is quite different from that of mainsequence stars. For instance, gravity is nearly constant on the 
Table 1. Characteristic times.

\begin{tabular}{llcccccc}
\hline \hline$T_{\text {eff }}$ & {$[\mathrm{K}]$} & 26300 & 14606 & 13196 & 11297 & 10586 & 9365 \\
$M / M_{\odot}$ & & 0.478 & 0.550 & 0.560 & 0.570 & 0.580 & 0.587 \\
$R$ & {$\left[10^{10} \mathrm{~cm}\right]$} & 1.292 & 5.325 & 6.728 & 10.12 & 13.77 & 17.44 \\
$\log g$ & {$\left[\mathrm{~cm} \mathrm{~s}^{-2}\right]$} & 5.58 & 4.41 & 4.22 & 3.87 & 3.61 & 3.41 \\
\hline$M_{\mathrm{zc}} / M_{\odot}$ & & $1.0 \times 10^{-12}$ & $3.4 \times 10^{-10}$ & $8.6 \times 10^{-10}$ & $4.0 \times 10^{-9}$ & $1.2 \times 10^{-8}$ & $2.9 \times 10^{-8}$ \\
$\rho\left(R_{\mathrm{zc}}\right)$ & {$\left[10^{-8} \mathrm{~g} \mathrm{~cm}^{-3}\right]$} & 5.3 & 4.5 & 4.3 & 3.9 & 3.5 & 3.9 \\
$u_{\mathrm{g}}\left(R_{\mathrm{zc}}\right)$ & {$\left[10^{-5} \mathrm{~cm} \mathrm{~s}^{-1}\right]$} & -16.3 & -1.24 & -0.832 & -0.405 & -0.244 & -0.139 \\
$R_{\mathrm{zc}}$ & {$\left[10^{10} \mathrm{~cm}\right]$} & 1.289 & 5.29 & 6.65 & 9.89 & 13.31 & 16.7 \\
\hline$\tau$ & {$\left[10^{6} \mathrm{yr}\right]$} & 0.0035 & 1.09 & 2.73 & 13.0 & 39.8 & 96.2 \\
\hline
\end{tabular}

ZAMS as $T_{\text {eff }}$ and $M_{*}$ vary, but on the ZAHB, $T_{\text {eff }}$ and $\log g$ both vary while the mass varies only slightly.

In the case of $\mathrm{HgMn}$ stars, isotopic anomalies of $\mathrm{Hg}$ have been observed (Preston 1971). The only model yet suggested to explain these involves diffusion in the atmosphere itself (Michaud et al. 1974). So the disappearance of the He convection zone is apparently essential to explain their properties. No such isotope anomaly of $\mathrm{Hg}$ has yet been observed on $\mathrm{HB}$ stars so that the critical velocity we determine by the disappearance of the He convection zone may also be viewed as a critical velocity for diffusion processes to dominate over the mixing caused by meridional circulation (see also Charbonneau \& Michaud 1991).

The remainder of this paper is organized as follows. In Sect. 2, the mathematical and physical descriptions of the transport problem are formulated in the context of HB stars, including a discussion of the calculation of meridional circulation flow solutions appropriate for these objects. Section 3 presents a series of numerical results carried out in a suite of ZAHB models spanning the horizontal branch. These parameter-free calculations yield a determination of the transitional effective temperature above which strong abundance anomalies can be expected, a result that turns out in good agreement with observations. We conclude in Sect. 4 with a brief critical discussion of modeling assumptions, and of the extent to which the settling of He may be viewed as a measure of the development of metal overabundances.

\section{Mathematical and physical formulation}

As in most stellar abundance evolution modeling we assume that spectroscopically determined photospheric abundances characterize the chemical composition of the star's outer convection zone. In other words, the convective envelope is thoroughly mixed on very short timescales by thermally-driven turbulent fluid motions. The surface abundances thus vary in response to mixing and/or chemical separation processes acting below the base of the convection zone, and (possibly) accretion or mass loss taking place in the outer surface layers.

\subsection{Stellar models}

Five of the six HB stellar models (see Table 1) used for this study were obtained as described in Michaud et al. (2007). For this study, models were calculated neglecting atomic diffusion with a metallicity of $Z=10^{-3}$. The Rosseland opacity was continuously recalculated for all species included in OPAL (Iglesias \& Rogers 1991, 1993, 1995, 1996; Rogers \& Iglesias 1992a,b), at each time step and every grid point, as the composition changed during the evolution from the zero age main-sequence to the
HB. Calculations were carried out in detail from the ZAMS to the He flash using the same code as described in Richard et al. (2002) and Richer et al. (1998). When the energy from $\mathrm{He}$ burning reached $10^{6} L_{\odot}$, the evolution on the red giant branch (RGB) was stopped. A fraction of the envelope mass was removed and the star was reconverged on the HB following essentially the procedure used by Sweigart (1987) and Dorman et al. (1991), and briefly described as Method 2 by Serenelli \& Weiss (2005). The five stars have the same core mass, $M_{\mathrm{c}}=0.4845 M_{\odot}$, but a slightly different envelope mass. The sixth model (with $T_{\text {eff }}=26300 \mathrm{~K}$ ) is a EHB model kindly communicated to us by B. Dorman (see Dorman et al. 1993, for details of this model). Shortly after each evolutionary model reached the ZAHB as defined in Michaud et al. (2007), a structure model was extracted and used for calculations with meridional circulation as described in the following subsections.

\subsection{Chemical separation and mixing: the transport equation}

Let $c(r, \theta, t)$ represent the concentration, at fractional radius $r$ and colatitude $\theta$, of a trace species mixed with a background of ionized hydrogen, in other words, the ratio $(\ll 1)$ of the number density of a trace species to protons ${ }^{1}$. In the present context it evolves according to the following two-dimensional linear advection-diffusion equation (Charbonneau \& Michaud 1988, 1991):

$\rho \frac{\partial c}{\partial t}+\rho \boldsymbol{U} \cdot \nabla c=\nabla \cdot\left(\rho D \nabla c-\rho \boldsymbol{u}_{g} c\right)$,

where $\rho$ is the mass density and $D$ is the total diffusion coefficient (more on this shortly), both only a function of radius $r$ in the stellar model (spherical polar coordinates are used throughout) since we do not consider the evolution of the star. The flow $\boldsymbol{U}$ is a specified steady, large-scale axisymmetric meridional circulation, i.e.,

$\boldsymbol{U}(r, \theta)=u_{r}(r, \theta) \hat{\boldsymbol{e}}_{r}+u_{\theta}(r, \theta) \hat{\boldsymbol{e}}_{\theta}$.

Equation (2) is nothing more than a statement of mass conservation for the trace species: in any given volume element, a time variation of the local concentration (first term on LHS) is due to either material being bodily advected (second term on the LHS) or diffusing (term on the RHS) in or out of the volume. Note that "diffusion" includes here both a diffusion term (first term within parentheses on the RHS of Eq. (2)), as well as a drift

\footnotetext{
${ }^{1}$ For the meridional circulation calculations, $\mathrm{He}$ is treated as a trace species. However the trace species approximation is not made in the stellar evolution calculations described in Michaud et al. (2007).
} 
velocity term, $\boldsymbol{u}_{g}$. In general the latter includes chemical separation effects from gravitational settling, radiative acceleration, and differential thermal diffusion:

$\boldsymbol{u}_{\mathrm{g}}(r)=D_{12}\left[(1-2 A+Z) \frac{m_{\mathrm{p}} g}{2 k T}+\frac{A m_{\mathrm{p}} g_{\mathrm{rad}}}{k T}-k_{T} \frac{\mathrm{d} \ln T}{\mathrm{~d} r}\right] \hat{\boldsymbol{e}}_{r}$,

where $D_{12}$ is the microscopic diffusion coefficient, $k_{\mathrm{T}}$ is the thermal diffusion coefficient, and $g_{\mathrm{rad}}$ is the radiative acceleration. The latter is negligible in the case of He, and is omitted in the calculations reported upon below. All other symbols have their usual meaning.

The total diffusion coefficient $D$ in Eq. (2) is assumed to include two contributions, namely microscopic diffusion and an enhanced "turbulent" diffusion acting only within the convective envelope:

$D(r)= \begin{cases}D_{\mathrm{T}} & \text { for } r \geq r_{\mathrm{CZ}} \\ D_{12}(r) & \text { otherwise. }\end{cases}$

This is a computationally convenient way to mimic the efficient mixing associated with turbulent flows within the thin, outer convective envelope of HB stars. The microscopic diffusion coefficient $D_{12}$ is computed according to the procedure described in Paquette et al. (1986), and the value of $D_{\mathrm{T}}$ is chosen high enough to ensure full mixing within the convective envelope on a timescale much shorter than all other relevant timescales in the problem. In the calculations reported below, a value

$D_{\mathrm{T}}=10^{4} \times D_{12}$

was found to suffice. The resulting discontinuity in $D$ occurring at the base of the superficial convection zone is readily handled within a finite element-based numerical integration scheme (more on the latter further below).

\subsection{Meridional circulation}

In what follows, we use, for $\boldsymbol{U}$, meridional circulation profiles recomputed using the general procedure outlined in Tassoul \& Tassoul (1982, hereafter TT82) for the thermally-driven circulatory flow arising inevitably in the radiative interior of rotationally distorted stars, as a consequence of the impossibility to achieve simultaneously radiative and hydrostatic equilibria (the so-called von Zeipel paradox). The TT82 procedure involves expanding all structure variables in power series of the small dimensionless parameter

$\varepsilon=\frac{v_{\mathrm{e}}^{2} R}{G M}$

where $v_{\mathrm{e}}$ is the star's equatorial rotational velocity. For a star rotating as a rigid or near-rigid body, it can be shown that to first order in $\varepsilon$, the meridional flow takes the form of quadrupolar cells, with radial and latitudinal flow components given by:

$u_{r}(r, \mu)=\varepsilon u(r) P_{2}(\mu)$,

$u_{\mu}(r, \mu)=\varepsilon v(r)\left(1-\mu^{2}\right) \frac{\mathrm{d} P_{2}(\mu)}{\mathrm{d} \mu}$

with $\mu=\cos \theta, u_{\theta}=-r u_{\mu} / \sin \theta$, and $P_{2}(\mu)=\left(3 \mu^{2}-1\right) / 2$ is the second order Legendre polynomial. The radial variations of the two flow components, embodied in the two functionals, $u(r)$ and $v(r)$, are constrained by the requirement of mass conservation $(\nabla \cdot \rho \boldsymbol{u}=0)$ to satisfy

$v(r)=\frac{1}{6} \frac{1}{\rho r^{2}} \frac{\mathrm{d}}{\mathrm{d} r}\left(\rho r^{2} u\right)$

so that the form of the circulatory flow is entirely set by $u(r)$, itself a function of the star's structure.

TT82 provide tabulations of $u(r)$ for two polytropic stellar structure models; these provide adequate representations of unevolved early-type main-sequence stars, but are less appropriate for HB stars. We therefore recomputed $u(r)$ for each of the HB structure models described in Sect. 2.1 above.

In the bulk of the radiative interior of a rotating star, the functional $u(r)$ is given by

$u_{\mathrm{S}}(r)=\frac{2 L r^{4}}{G^{2} m^{3}} \frac{n+1}{n-3 / 2}\left[h^{\prime}+\left(\frac{2}{r}-\frac{m^{\prime}}{m}\right) h\right]$,

where $n$ is the polytropic index, corresponding to the ratio

$n=\frac{\rho^{\prime} T}{\rho T^{\prime}}$,

and with

$m^{\prime}=4 \pi \rho r^{2}$

$h=A \Phi(r)$

$A=\frac{1}{3} \omega_{0}^{2} \frac{5 R^{2}}{3 \Phi(R)+R \Phi^{\prime}(R)}$,

where primes denote differentiation with respect to radius. The potential $\Phi$ is a solution of

$\frac{\mathrm{d}^{2} \Phi}{\mathrm{d} r^{2}}+\frac{2}{r} \frac{\mathrm{d} \Phi}{\mathrm{d} r}-\frac{6}{r^{2}} \Phi+4 \pi G \frac{\rho \rho^{\prime}}{p^{\prime}} \Phi=0$

subject to the boundary conditions:

$\Phi(0)=0, \quad \Phi^{\prime}(0)=0$

(see Sect. IV of TT82 and references therein). The reconstruction procedure begins with numerical integration of Eq. (16) using tabulated values for the pressure $p$ and density $\rho$ from the HB structure model. Working back from Eq. (15) up through (11) eventually yields $u_{\mathrm{S}}(r)$, with the required radial derivatives being evaluated using second-order centered finite differences.

The $u_{\mathrm{S}}(r)$ profiles resulting from reconstruction are shown in Fig. 2, for our six HB models, as labeled. The gray lines show alternate profiles constructed by direct rescaling of the $3 M_{\odot}$ ZAMS model solutions tabulated in Sect. VI of TT82. While these provide tolerable approximations in the bulk of the radiative envelope, significant differences with the solutions reconstructed here are apparent, especially near the surface layers. These differences can be traced to radial variations in Rosseland opacities resulting from our use of OPAL opacities, and are absent from the Cowling point source models with Kramer's opacity law used in TT82. These opacity variations alter the stratification in the outer envelope, and feed back on the $u_{\mathrm{S}}(r)$ profile mainly through the computed value of effective polytropic index (Eq. (12)) via the $T / T^{\prime}$ radial dependency. We verified, in the $0.587 M_{\odot}$ model, that the shape of the $u_{\mathrm{S}}(r)$ curve for $r>0.8 R_{*}$ closely mimics that of $\log \kappa_{\mathrm{R}}$. These radial variations distort the 


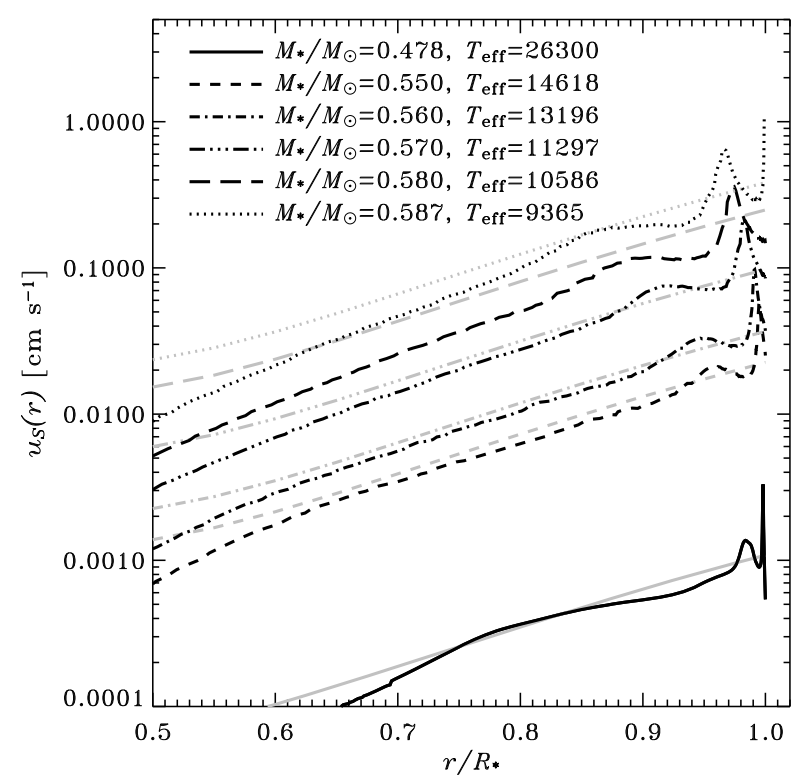

Fig. 2. Functional $u_{\mathrm{S}}(r)$ defining the meridional flow in the bulk of the interior, for the six ZAHB structure models used in this study. The gray curves are the corresponding, approximate solutions that can be obtained by a simple rescaling of the solutions tabulated in TT82 (see text).

shape of the meridional flow cell in the outer reaches of the radiative envelope, but the overall pattern remains quadrupolar, with one cell per meridional quadrant.

Another trend immediately apparent in Fig. 2 is the overall systematic increase of $u_{\mathrm{S}}(r)$, at any given depths, with decreasing surface temperature. This arises primarily through the stellar radius variations, which enter Eq. (11) as a $r^{4}$ dependency, leading to a $g^{-2}$ dependency. Other stellar model parameters such as mass or luminosity, have here a much lesser impact on the magnitude of $u_{\mathrm{S}}(r)$.

Equation (11) diverges at the boundaries of convection zones (where $n=3 / 2$ ), and yields diverging latitudinal meridional flow velocities at the stellar surface $(\rho \rightarrow 0$ when evaluating Eq. (9) using Eq. (10)). Tassoul \& Tassoul (1982) corrected these shortcomings by computing boundary layer solutions that behave properly at the surface or at radiative/convective boundaries, and that are smoothly matched onto the interior solution described by Eq. (11). We follow here the same procedure, using the appropriate dimensionless boundary layer solutions, as tabulated in TT82.

Our HB stellar models do include a thin convective layer in their outer radiative envelope. How much mixing effectively takes place between these layers and the underlying meridional flow is one of the primary uncertainties in the present abundance evolution calculations. One possibility is to close the meridional flow immediately beneath this convective layer, using the core boundary layer solution described and tabulated in Sect. IVa of TT82. One then has $u_{r}=0$ at the base of the convective layer, implying minimal impact of the meridional flow on He settling ${ }^{2}$. Alternately, one could assume, as in Charbonneau \& Michaud (1988), that the meridional flow effectively penetrates the convective envelope, thus competing more directly with He settling

2 That an impact does remain is due to the fact that circulation will greatly affect the abundance and radial abundance gradient immediately beneath the base of the convection zone, and thus He transport by microscopic diffusion. out of the convective layers. We then use the surface boundary layer solution of TT82 (Sect. IVb) to close the meridional flow at the stellar surface. These represent the two possible extreme cases regarding the influence of the meridional flow on the gravitational settling of $\mathrm{He}$, and, in what follows, abundance evolution calculations are carried out for these two extreme cases, as they effectively bracket the range of intermediate possibilities.

\subsection{Numerical solution}

Equation (2) defines a linear initial-boundary-value problem in two spatial dimensions $[r, \theta]$. While this may at first glance seem to be an entirely straightforward partial differential equation to solve numerically, it actually offers some substantial practical difficulties. The wide range of timescales characterizing the various physical processes at play renders impractical the use of explicit time-stepping. Furthermore, the "slow" meridional flow is still "fast" from the computational point of view, as its associated grid Peclet number is very much larger than unity, which in turn imposes the use of a relatively dense spatial mesh in order to ensure numerical stability and accuracy. Finally, the desired integration time (up to $10^{7} \mathrm{yr}$ ) is very much larger than chemical separation timescales in the problem (as low as a few $10^{3} \mathrm{yr}$ in the hotter models).

Starting from uniform He abundances throughout the radiative envelope on the $\mathrm{ZAHB}^{3}$ ( $t=0$ in the simulations), Eq. (2) is solved numerically using a standard time-implicit finite element method, more specifically the scheme described in Charbonneau \& Michaud (1991), but with upwinding turned off so as to minimize numerical diffusion. Homogeneous initial He abundances throughout the radiative envelope and convection zone are used as initial condition. Mass loss and accretion are neglected, so that the surface boundary condition is simply zero-flux since $u_{r}=0$ at $r / R=1$. As discussed earlier, the outer convection zone remains thouroughly mixed throughout the evolution via an appropriate numerical choice for the "turbulent" diffusion coefficient $D_{\mathrm{T}}$ (cf. Eq. (5)).

\section{Results}

\subsection{Characteristic timescales}

Prior to launching into full-blown numerical calculations, it is instructive to estimate typical abundance evolution timescales for He settling in the absence of competing mixing processes. Under these circumstances, the outer convection zone simply acts as a reservoir draining from below at a rate set by the He gravitational settling velocity, hereafter denoted $u_{\mathrm{g}}$. Knowing the magnitude of this velocity and of the density at the base of the convection zone, and the mass $M_{\mathrm{cz}}$ contained in the latter, one can estimate an $e$-folding timescale $\tau$ for the convection zone He abundance:

$$
\tau=\left.\frac{M_{\mathrm{cz}}}{\rho u_{\mathrm{g}} 4 \pi r^{2}}\right|_{r=R_{\mathrm{cz}}} .
$$

This timescale is listed in the bottom row of Table 1 for our six HB models. The pronounced decrease of $\tau$ in going from the cooler to the hotter model is primarily a consequence of the rapidly decreasing convection zone mass (fifth line in the table); since the $T$ is very nearly the same in all models at the base of the He II convection zone $\left(T_{\mathrm{CZ}} \sim 50000 \mathrm{~K}\right)$, the denominator turns out to be nearly the same in all models. In all but our coolest

\footnotetext{
3 The He abundance variation in the thin $\mathrm{H}$ burning shell is neglected.
} 

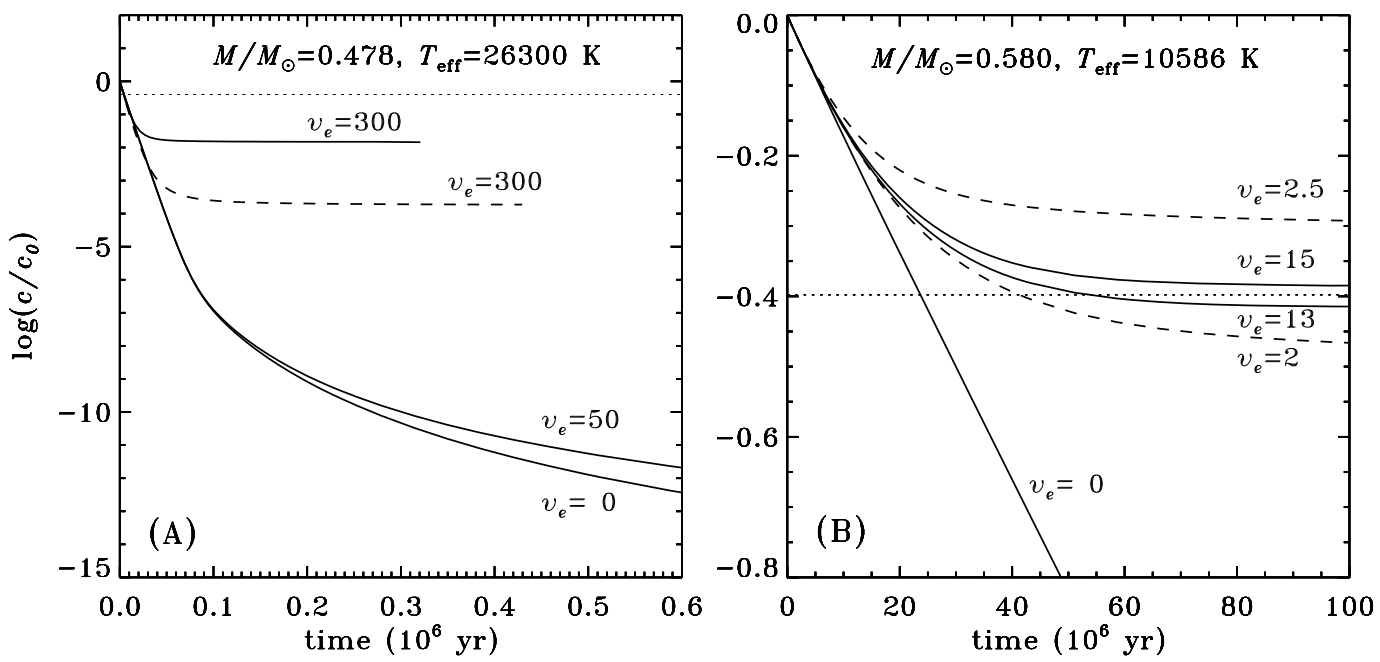

Fig. 3. Evolution of He abundance in the superficial convection zone of $\mathrm{HB}$ star models with effective temperatures $T_{\text {eff }}=26300$ and $10586 \mathrm{~K}$ (panels $\mathbf{A}$ and $\mathbf{B}$, respectively). The curves are labeled in terms of the assumed equatorial rotational velocity, in $\mathrm{km} \mathrm{s}^{-1}$. Dashed curves correspond to meridional circulation penetrating the convection zone; solid curves corresponds to circulation streamlines closing in a boundary layer immediately beneath the convection zone. The horizontal dotted line indicates the critical He concentration below which the He convection zone disappears (see text).

model, the chemical separation timecale is much shorter than the HB lifetime (of order $10^{8} \mathrm{yr}$ ), which justifies the use of static stellar models for the upcoming numerical calculations.

Generally speaking, the marked decrease of the chemical separation timescale $\tau$ with increasing $T_{\text {eff }}$ is certainly compatible with the fact that strong anomalies are observed in hotter HB stars, but leaves unexplained the sharp bimodality in abundance pattern observed across the $T_{\text {eff }} \simeq 11000 \mathrm{~K}$ boundary (cf. Fig. 1 herein). As with HgMn stars, this is where the competition between He settling and mixing by meridional circulation enters the picture.

\subsection{Critical equatorial rotational velocity}

Using the numerical procedure described in the preceeding section, we simulated the spatiotemporal evolution of the He abundance in the radiative envelope and outer convective layers of the six HB models whose characteristics are listed in Table 1. The simulations were carried out for various equatorial rotational velocities and two treatments of meridional circulation at the base of the superficial convection zone. Note that once the latter has been chosen, the solution of Eq. (2) involves no adjustable parameter.

Figure 3 shows a sample of representative evolution curves for the $\mathrm{He}$ abundances in the (mixed) convective layers in two $\mathrm{HB}$ models, computed using equatorial rotational velocities as labeled (in $\mathrm{km} \mathrm{s}^{-1}$ ). Figure $3 \mathrm{~A}$ shows results for our hottest $\mathrm{HB}$ model $\left(T_{\text {eff }}=26300 \mathrm{~K}\right)$, while Fig. $3 \mathrm{~B}$ corresponds to one of our cooler HB models $\left(T_{\text {eff }}=10586 \mathrm{~K}\right)$. Dashed lines correspond to cases where meridional circulation penetrates the convection zone, while solid lines show solutions where the circulation closes within a thin boundary beneath the base of the convection zone. Note that even in this case, circulation can have a measurable effect on the evolution of He abundances within the convection zone, as it alters the concentration gradients at its base. Morphologically similar curves are obtained in our other four HB models.

In the absence of a competing mechanism, gravitational settling leads to an exponential decrease in He abundance, on timescales in good agreement with the estimates made in
Sect. 3.1. With circulation present, material undepleted in $\mathrm{He}$ is continuously being advected into the polar regions of the convection zone from below, while in equatorial regions circulation accelerates He depletion. A state of equilibrium is eventually reached, characterized by vanishing total He flux at the base of the convection zone. From that point on, the He abundance in the convective envelope ceases to decrease. The stronger the meridional circulation, the higher the He equilibrium abundance, a pattern readily visible in Fig. 3 (recall that the meridional flow velocity scales as $v_{\mathrm{e}}^{2}$, as per Eqs. (7)-(9)). This is the same type of equilibrium state characterizing the calculations of Charbonneau \& Michaud (1988) for main-sequence HgMn stars, and indeed the curves in Fig. 3 herein are morphologically similar to those characterizing $\mathrm{He}$ settling in $\mathrm{HgMn}$ stars in the presence of meridional circulation.

If this equilibrium abundance in the convection zone remains higher than $0.4 \times$ solar (corresponding here to a depletion factor of 0.4 dex, shown as a dotted line in Fig. 3), its contribution to the total opacity remains large enough to sustain thermallydriven convection. Otherwise, convection ceases in the lower part of the convection zone, and chemical separation can proceed much closer to the stellar surface. One therefore expects stronger abundance anomalies of other chemical species in this latter case than in the former. This is the essence of the explanation proposed by Michaud (1982) for the observed upper limit on rotational velocities observed in $\mathrm{HgMn}$ stars and vindicated by detailed numerical calculations in Charbonneau \& Michaud (1988). It is being extended here as a test of the importance of atomic diffusion in HB stars.

Figure 3 already shows that for the hotter models, where the settling velocities are quite high at the base of the (thin) convective envelope, excessively high rotational velocities are required to stop He settling; in fact, already at $v_{\mathrm{e}}=300 \mathrm{~km} \mathrm{~s}^{-1}$ the assumptions under which the TT82 meridional circulation are derived are probably no longer valid. However, in the cooler models (cf. Figs. 3A and B) rotational velocities commensurate with observed values are sufficient to prevent He settling. This is because the cooler the model, the larger the mass of its outer convective envelope (see also Table 1). For each HB star model it is therefore possible, from results such as shown 


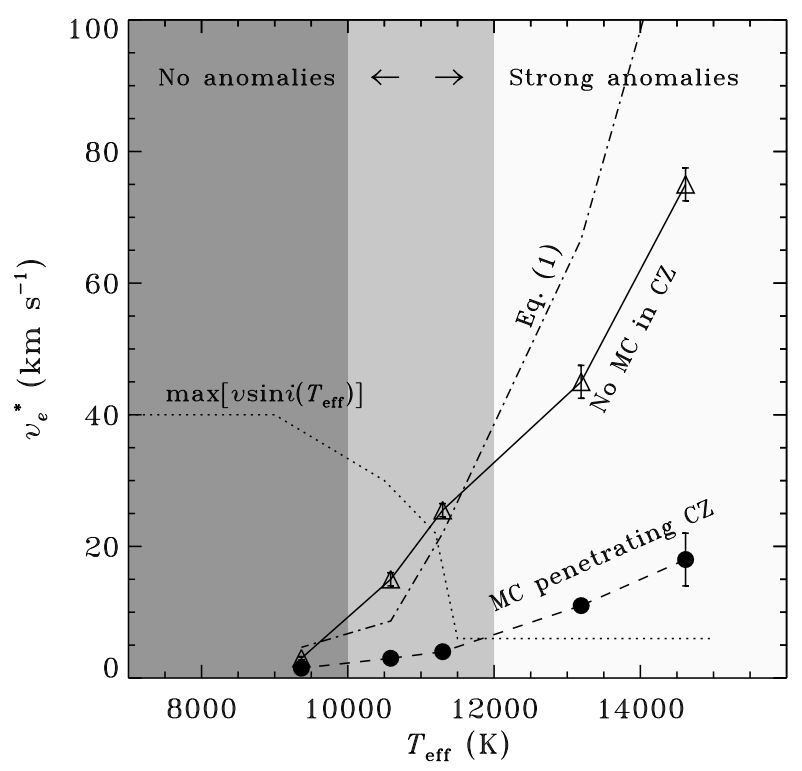

Fig. 4. Critical equatorial rotational velocity, $v_{\mathrm{e}}^{*}$, versus effective temperature, $T_{\text {eff }}$, for our six HB model sequence. Solid dots correspond to calculations where meridional circulation penetrates the convection zone, while open triangles refer to the opposite extreme possibility, where circulation closes within a boundary layer immediately beneath the base of the convective envelope, without any penetration therein. The dotted line indicates the upper envelope of observed $v \sin i$ values of Fig. 1 (lower panel), while the shading refers to the observed presence (white) or absence (dark gray) of strong abundance anomalies. The intermediate shade of gray defines an interval where both anomalies are present only in a fraction of stars. The dot-dashed line gives the critical velocity $v_{\mathrm{e}}^{*}$ according to Eq. (1), using the value $v_{\mathrm{e}}^{*}=100 \mathrm{~km} \mathrm{~s}^{-1}$ for $15000 \mathrm{~K}$ and $\log g=4.4$ to set the proportionality constant.

in Fig. 3, to estimate the critical equatorial velocity $\left(v_{\mathrm{e}}^{*}\right)$ delineating these two regimes. For example, Fig. 3B indicates that in our $T_{\text {eff }}=10586 \mathrm{~K} \mathrm{model,} v_{\mathrm{e}}^{*} \simeq 14 \pm 1 \mathrm{~km} \mathrm{~s}^{-1}$ if the circulation closes at the base of the convective envelope, but that $v_{\mathrm{e}}^{*} \sim 2.2 \mathrm{~km} \mathrm{~s}^{-1}$ if penetration of the meridional flow is allowed.

Figure 4 summarizes the results of carrying out this exercise for our six HB models and two working assumptions regarding penetration of meridional circulation in the convection zone. Critical rotational velocities are plotted as a function of effective temperature, for circulation penetrating the convection zone (solid dots), or closing at its base (open triangles) ${ }^{4}$. The dotted line gives the upper envelope of observed $v \sin i$ values at a given $T_{\text {eff }}$, constructed from data presented in Fig. 23 of Behr (2003a), Fig. 8 of Behr (2003b), and Fig. 5 of Recio-Blanco et al. (2004). The gray shading indicates the presence or absence of abundance anomalies: dark gray indicates normal metallic abundances, and white the $T_{\text {eff }}$ domain where metal overabundances are observed. The light gray region, corresponding to $10000 \leq T_{\text {eff }} \leq 12000 \mathrm{~K}$, is where the observational picture is not so clear cut, with HB stars showing significant abundance anomalies in some globular clusters but not in others. Figure 1 shows the data for a sample of the clusters.

Figure 4 should be "read" as follows: at a given $T_{\text {eff }}$, if the critical rotation curve lies above the dotted line, then mixing

\footnotetext{
4 Since these two cases represent the extremes in the possible penetration of meridional circulation in the outer convection zone, and since reality probably lies at some ill-determined locus somewhere in between, no particular effort was made to determine these critical rotational velocities to better than two significant figures.
}

by rotationally-induced meridional circulation cannot compete with He settling even in the fastest rotators, which should then lead to the disappearance of a good portion of the outer convection zone, with concomittent buildup of photospheric metallic abundance anomalies by chemical separation. In comparing our modeling results to observations, a key feature is the intersection of the two critical rotational velocity curves with the upper envelope of observed $v \sin i$ values. For circulation closing at the base of the convective envelope, this takes place at $T_{\mathrm{eff}} \simeq 11000 \mathrm{~K}$, and at $11500 \mathrm{~K}$ if circulation is allowed to penetrate the convection zone. We use these two extreme cases for the treatment of meridional circulation at the base of the superficial convection zone to assign an uncertainty to our transition effective temperature: $T_{\text {eff }}=11500 \pm 250 \mathrm{~K}$. Our calculations would predict that for stars hotter than these values, meridional circulation cannot stop He settling, and strong abundance anomalies should develop. Given uncertainties in $T_{\text {eff }}$ scales and in $\sin i$, it is quite striking that the $T_{\text {eff }}$ range predicted by the calculations falls neatly within the effective temperature range $11000 \pm 500 \mathrm{~K}$ above which strong abundance anomalies start to be observed in HB stars. Recall, moreover, that this good agreement occurs without any adjustment of free-parameters, as the abundance evolution model does not contain any, being instead entirely set by the physical properties of the underlying stellar structure models. Comparing Figs. 1 and 4, one notes that, in the clusters shown, the only star with $T_{\text {eff }}>12000 \mathrm{~K}$ and normal abundances, M15/B348, has a $v \sin i \sim 20 \mathrm{~km} \mathrm{~s}^{-1}$, which is between the two critical rotational velocity curves. Stars between $11000 \mathrm{~K}$ and $12000 \mathrm{~K}$ that have abundance anomalies also have a $v \sin i$ lower than both critical rotation velocities in nearly all cases.

The dot-dashed line in Fig. 4 corresponds to the variations of the critical equatorial rotational velocity with $T_{\text {eff }}$ predicted by Eq. (1), anchored using the value $v_{\mathrm{e}}^{*}=100 \mathrm{~km} \mathrm{~s}^{-1}$ at $T_{\text {eff }}=$ $15000 \mathrm{~K}$ and $\log g=4.4$ corresponding to the case of $\mathrm{HgMn}$ stars. This approximate expression does provide a tolerable representation of the numerical results, yielding a transitional effective temperature of $T_{\text {eff }} \simeq 11500 \mathrm{~K}$ above which strong abundance anomalies are expected. The differences with the numerical results originate primarily from differences between the internal structures of our ZAHB stellar models and those of mainsequence stars of similar effective temperatures, including compositional differences, that affect chemical separation velocities as well as meridional flow profiles (cf. Fig. 2).

\section{Discussion and conclusion}

In this paper we have examined the competition between gravitational settling and mixing by a large-scale, thermally-driven meridional flow in the radiative envelope of HB stars. Focusing on the case of helium, our numerical results show that He settling should be sufficiently impeded for $T_{\text {eff }}<11500 \pm 250 \mathrm{~K}$ to prevent the disappearance of the superficial convection zone. For ZAHB stellar models, this leads to the conclusion that strong anomalies should be restricted to $T_{\text {eff }}>11500 \pm 250 \mathrm{~K}$. In a transitional interval (11000 to $12000 \mathrm{~K})$ one sees anomalies in the less rapidly rotating stars only.

The numerical calculations of Charbonneau \& Michaud (1991), examining the competition between chemical separation and transport by meridional circulation in FmAm stars, showed that, in the absence of other competing processes such as mass loss or turbulent mixing, underabundant chemical species are typically far more sensitive than overabundant species to the mixing effects of meridional circulation (see their Fig. 6). For 
some species, overabundances are expected even if a star rotates more rapidly by a factor of 10 than the critical velocity. A detailed calculation for metals is outside the scope of this paper. When a star rotates more slowly than the critical velocity, overabundances of metals shoud definitely appear (see Michaud et al. 2008, for the calculation of the anomalies of metals to be expected in HB stars). However one would also expect that, in the $T_{\text {eff }}>11000 \mathrm{~K}$ regime, the magnitude of underabundances should show an anticorrelation with $v \sin i$. Some candidate elements to test this expectation are $\mathrm{C}$ and $\mathrm{O}$ in addition to $\mathrm{He}$. In this context, it would be particularly interesting to compare in detail chemical abundances in the relatively rapidly rotating $\mathrm{HB}$ star M15/B348 to those of its more slowly rotating counterparts with similar $T_{\text {eff }}$.

Detailed abundance modeling, including structural evolutionary effects, could then possibly constrain to some degree meridional circulation models, and in particular the extent of hydrodynamical coupling with the superficial convection zone of HB stars.

The results of our abundance evolution calculations are predicated on the use of a specific model, namely that of TT82, for the meridional circulation in the radiative envelope of a slowly rotating star. Other circulation models have been proposed, notably the so-called "shellular" solutions of Zahn (1992, hereafter Z92; see also Mathis \& Zahn 2004). It is instructive to contrast the $a$ priori assumptions made in these two distinct modeling frameworks. TT82 assume the presence of turbulence in the radiative interior, isotropic and with a turbulent viscosity some 3 orders of magnitude larger than ordinary microscopic viscosity. This is sufficient to maintain a state of near-rigid rotation despite angular momentum advection by the meridional flow, thus producing an internally consistent solution. Z92 also invokes turbulence, but strongly anisotropic, with the horizontal viscosity exceeding the microscopic values by some 8 orders of magnitude, the exact value being usually treated as a free parameter in evolutionary calculations. The Z92 solutions are thus characterized by an angular velocity developing radial gradients in response to the advection of angular momentum by circulation. The latter reduces to a diffusive-like transport plus a purely radial advective contribution that, conveniently, can both be accomodated within a 1D formalism. TT82 find support in their turbulence Ansatz in various geophysical flows, where effective viscosities can be measured and are indeed often found to be 3-4 orders of magnitude above microscopic. Z92 finds support for his views in some laboratory experiments, as removed from stellar interior conditions as TT82's geophysical flows, and the (undeniable) fact that stellar radiative interior are strongly stratified. Interestingly, for stars not subjected to significant angular momentum loss, circulation patterns computed using the Z92 formalism (see, e.g., Mathis et al. 2007, Fig. 2) closely resemble the TT82 solutions in the outer part of the radiative envelope, significant differences being restricted to the vicinity of the convective core.

At any rate, within the TT82 modeling framework adopted here, the transport model for the competition between He settling and advection by meridional circulation is entirely parameterfree, once a treatment of meridional circulation at the base of the superficial convection zone has been adopted. The latter is the primary internal uncertainty affecting the present calculations. As can be seen in Fig. 4, this leads to an uncertainly of about $500 \mathrm{~K}$ on the effective temperature above which strong anomalies should begin to materialize. The transport model, of course, leaves the origin of the $v \sin i$ bimodality entirely unexplained; all we have shown is that given the existence of this rotational bimodality, the corresponding bimodality in abundance anomalies arises naturally as a consequence of the competition between the advection by meridional circulation and the gravitational settling of helium.

It appears most likely that the rotational bimodality has originated prior to the HB phase, and could have arisen in a number of ways (see the discussion in Behr et al. 2000b, and references therein). A very interesting clue is provided by the $\mathrm{sdO}$ and hot sdB subwarfs $\left(T_{\text {eff }}>30000 \mathrm{~K}\right)$, which collectively distribute themselves in the HR diagram as a hot extension of the horizontal branch. Although their evolutionary relationship to the hot $\mathrm{HB}$ stars remains uncertain, the $\mathrm{sdO}$ and sdB subdwarfs also burn Hein their center, are also slow rotators, and their observed abundances show signatures of chemical separation in the presence of a weak mass loss at a rate $\sim 10^{-15}-10^{-14} M_{\odot} \mathrm{yr}^{-1}$ (Michaud et al. 1985). Such mass loss rates are well below the radiatively-driven mass loss rates that Unglaub (2008) obtained for hot subdwarfs 5 . Even more remarkable is the fact that all recent attempts made at measuring magnetic fields in hot subdwarfs have yielded positive detections (see Elkin 1996; O'Toole et al. 2005; Valyavin et al. 2006). Could this ubiquitous magnetism extend to cooler sdBs, or even all the way down to $\sim 11000 \mathrm{~K}$ along the HB? Could magnetism be the cause of the observed slow rotation of hot HB stars? The link between magnetism, mass loss and rotation is well established for mainsequence stars in terms of angular momentum loss mediated by magnetized winds (e.g., Weber \& Davis 1967; Mestel 1968), with magnetic fields also remaining the most promising mechanism for angular momentum redistribution within radiative interiors (Mestel \& Weiss 1987; Charbonneau \& MacGregor 1993; Spruit 2002; Denissenkov \& Pinsonneault 2007; Denissenkov et al. 2008). Could magnetism play a major role in hot $\left(T_{\mathrm{eff}}>\right.$ $11500 \mathrm{~K}) \mathrm{HB}$ stars? It also plays a major role for abundance anomalies in most magnetic Ap-Bp stars but apparently not at the surface of HgMn stars whose slow rotation is currently unexplained. Mestel (1999) however suggested (his page 176) that an interior magnetic field might force near isorotation and so explain the success of the meridional circulation model in explaining abundance anomalies on $\mathrm{HgMn}$ stars without the requirement for shear turbulence.

Acknowledgements. This work was supported via NSERC Discovery grants to P.C. and G.M.

\section{References}

Abt, H. A., Levato, H., \& Grosso, M. 2002, ApJ, 573, 359

Babel, J. 1996, A\&A, 309, 867

Behr, B. B. 2003a, ApJS, 149, 67

Behr, B. B. 2003b, ApJS, 149, 101

Behr, B. B., Cohen, J. G., McCarthy, J. K., \& Djorgovski, S. G. 1999, ApJ, 517, L135

Behr, B. B., Cohen, J. G., \& McCarthy, J. K. 2000a, ApJ, 531, L37

Behr, B. B., Djorgovski, S. G., Cohen, J. G., et al. 2000b, ApJ, 528, 849

Charbonneau, P., \& MacGregor, K. B. 1993, ApJ, 417, 762

Charbonneau, P., \& Michaud, G. 1988, ApJ, 327, 809

Charbonneau, P., \& Michaud, G. 1991, ApJ, 370, 693

Denissenkov, P. A., \& Pinsonneault, M. 2007, ApJ, 655, 1157

Denissenkov, P. A., Pinsonneault, M., \& MacGregor, K. B. 2008, ApJ, 684, 757

Dorman, B., Lee, Y.-W., \& Vandenberg, D. A. 1991, ApJ, 366, 115

Dorman, B., Rood, R. T., \& O'Connell, R. W. 1993, ApJ, 419, 596

Elkin, V. G. 1996, A\&A, 312, L5

\footnotetext{
${ }^{5}$ His mass loss rates are probably upper limits because of his neglect of the shadowing caused by photospheric lines as discussed by Babel (1996).
} 
Fabbian, D., Recio-Blanco, A., Gratton, R. G., \& Piotto, G. 2005, A\&A, 434, 235

Glaspey, J. W., Demers, S., Moffat, A. F. J., \& Michaud, G. 1986, PASP, 98, 1123

Glaspey, J. W., Michaud, G., Moffat, A. F. J., \& Demers, S. 1989, ApJ, 339, 926

Gratton, R., Sneden, C., \& Carretta, E. 2004, ARA\&A, 42, 385

Iglesias, C. A., \& Rogers, F. J. 1991, ApJ, 371, L73

Iglesias, C. A., \& Rogers, F. J. 1993, ApJ, 412, 752

Iglesias, C. A., \& Rogers, F. J. 1995, ApJ, 443, 460

Iglesias, C. A., \& Rogers, F. J. 1996, ApJ, 464, 943

Jomaron, C. M., Dworetsky, M. M., \& Allen, C. S. 1999, MNRAS, 303, 555

Mathis, S., \& Zahn, J.-P. 2004, A\&A, 425, 229

Mathis, S., Decressin, T., Palacios, A., et al. 2007, Astron. Nachr., 328, 1062

Mestel, L. 1968, MNRAS, 138, 359

Mestel, L. 1999, Stellar magnetism (Oxford: Clarendon), International series of monographs on physics

Mestel, L., \& Weiss, N. O. 1987, MNRAS, 226, 123

Michaud, G. 1981, in Liege International Astrophysical Colloquia, Chemically Peculiar Stars of the Upper Main Sequence, ed. P. Renson (Liège: Université de Liège), 23, 355

Michaud, G. 1982, ApJ, 258, 349

Michaud, G. 1986, in Upper Main Sequence Stars with Anomalous Abundances, ed. C. R. Cowley, M. M. Dworetsky, \& C. Mégessier (Doldrecht: Reidel), IAU Coll., 90, 459

Michaud, G., Vauclair, G., \& Vauclair, S. 1983, ApJ, 267, 256

Michaud, G., Reeves, H., \& Charland, Y. 1974, A\&A, 37, 313

Michaud, G., Bergeron, P., Wesemael, F., \& Fontaine, G. 1985, ApJ, 299, 741

Michaud, G., Richer, J., \& Richard, O. 2004, in IAU Symp., 215, 457

Michaud, G., Richer, J., \& Richard, O. 2007, ApJ, 670, 1178
Michaud, G., Richer, J., \& Richard, O. 2008, ApJ, 675, 1223

Moehler, S. 2001, PASP, 113, 1162

Moehler, S., Sweigart, A. V., Landsman, W. B., \& Heber, U. 2000, A\&A, 360, 120

O’Toole, S. J., Jordan, S., Friedrich, S., \& Heber, U. 2005, A\&A, 437, 227

Pace, G., Recio-Blanco, A., Piotto, G., \& Momany, Y. 2006, A\&A, 452, 493 Paquette, C., Pelletier, C., Fontaine, G., \& Michaud, G. 1986, ApJS, 61, 177 Peterson, R. C. 1983, ApJ, 275, 737

Preston, G. W. 1971, ApJ, 164, L41

Recio-Blanco, A., Piotto, G., Aparicio, A., \& Renzini, A. 2002, ApJ, 572, L71 Recio-Blanco, A., Piotto, G., Aparicio, A., \& Renzini, A. 2004, A\&A, 417, 597

Richard, O., Michaud, G., Richer, J., et al. 2002, ApJ, 568, 979

Richer, J., Michaud, G., Rogers, F., et al. 1998, ApJ, 492, 833

Rogers, F. J., \& Iglesias, C. A. 1992a, ApJS, 79, 507

Rogers, F. J., \& Iglesias, C. A. 1992b, ApJ, 401, 361

Sargent, W. L. W., \& Searle, L. 1967, ApJ, 150, L33

Sargent, W. L. W., \& Searle, L. 1968, ApJ, 152, 443

Serenelli, A., \& Weiss, A. 2005, A\&A, 442, 1041

Spruit, H. C. 2002, A\&A, 381, 923

Sweigart, A. V. 1987, ApJS, 65, 95

Talon, S., Vincent, A., Michaud, G., \& Richer, J. 2003, J. Comp. Phys., 184, 244

Tassoul, J.-L., \& Tassoul, M. 1982, ApJS, 49, 317

Théado, S., \& Vauclair, S. 2003, ApJ, 587, 784

Unglaub, K. 2008, A\&A, 486, 923

Valyavin, G., Bagnulo, S., Fabrika, S., et al. 2006, ApJ, 648, 559

Weber, E. J., \& Davis, L. J. 1967, ApJ, 148, 217

Woolf, V. M., \& Lambert, D. L. 1999, ApJ, 521, 414

Yong, D., Grundahl, F., Johnson, J. A., \& Asplund, M. 2008, ApJ, 684, 1159

Zahn, J.-P. 1992, A\&A, 265, 115 